\title{
Design of a Pedestrian Detection System Based on OpenCV
}

\author{
Ning Xu and Yong Ren* \\ Applied Technology College of Soochow University \\ Suzhou, Jiangsu, 215325, P.R.China \\ Corresponding author: renyong@suda.edu.cn
}

Keywords: Pedestrian detection; OpenCV; Image process; Pattern recognition; Machine learning.

\begin{abstract}
Analysis on the background characteristics of combined pedestrian and road, pedestrian detection algorithm based on simulation and optimization of using OpenCV open source Visual Database design and implementation of a prototype system, the system can provide support and early warning for drivers, so as to minimize vehicle accidents from happening.
\end{abstract}

\section{Introduction}

According to statistics, by the end of 2012, and civilian cars in China reached 120.89 million units, up $14.3 \%$ from a year earlier, with private car ownership 93.09 million vehicles, an increase of $18.3 \%$. In 2011, the national road traffic accidents involving casualties amounted to 210812, 62387 people were killed. According to the traffic control Department statistics, due to traffic accidents, injuring one person out of every minute in China, China has become one of the highest traffic accident death toll. Because of bike riders and pedestrians in traffic accidents are often in a weak position, once the motor vehicle involved in a traffic accident, is vulnerable to injury. It can be said that pedestrians killed in traffic accidents are a major factor. Therefore, the study on intelligent vehicle pedestrian detection technology is a prominence in the front direction is one focus of object detection[1].

For pedestrian detection, scholars have long conducted in feature extraction, classification, and many other related research, which is represented by Paul Viola work[2]. In 2003, the Viola in the integral image method for fast computation of Haar features based on joint representation method using appearance and motion characteristics, and combining the series combination of sorting mechanisms, achieve better results in our pedestrian detection. Some scholars classify single parallel combination and synthesis of findings according to the test results of all classifications, such as Grubb with two SVM classifiers, respectively, for detection of pedestrians are on the back side, results of which were carried out between categories[3,4]. For the outside of the vehicle traffic information detection, including Lane, goals targets such as vehicles, obstacles and pedestrians, many scholars have proposed their own algorithms and system implementation[5].

Although pedestrian detection technology has achieved some results, but the requirement applied to autonomous driving of intelligent vehicles there is a relatively large gap, there are still some difficult problems need to be solved[6,7]. On one hand, intelligent vehicle systems with strong real-time requirements, you need to target for rapid detection of pedestrians on the other, by vehicle-mounted vision sensor to capture dynamic images are subject to many influences, such as weather and lighting conditions change, and, which makes it difficult to accurately detect the presence of pedestrians[8]. 


\section{Related Work}

With the development of machine vision and image processing technology, can take advantage of Visual understanding of technical driving help and tips to provide intuitive, thereby improving vehicle safety, vehicle based on machine vision aided system has become a research topic in the field of active safety.

This work is on the basis of previous research, comprehensive analysis and comparison of the methods of pedestrian detection through algorithm optimization and simulation to improve pedestrian detection algorithm of real-time, accurate, and using the open source computer vision library Open CV design and implementation of a prototype system[9]. Analysis of the system can be vehicle-mounted vision sensor to obtain the traffic image, goals such as rapid detection of pedestrians, thus perceiving the outside of the vehicle environment information, and assisted with the driver warning, minimize vehicle accidents from happening[10].

\section{System Design}

Effective to reduced traffic accident of occurred, can warned driver may and vehicles adjacent of obstacles, especially pedestrian occurred collision, now based on computer Visual of detection technology, due to pedestrian of wearing color each not same, and is easy and background confusion, objects of movement and external features also has differences, forecast by needed time also not too short, so intends to through optimization some algorithm, improve Executive efficiency, on using Visual information detection to of may contains pedestrian regional for judge, timely of warning driver[11]. In addition, full use of the multi-sensor data fusion to improve detection to determine. System has the following features:(1) high accuracy: changes in a variety of scenarios, such as sudden changes in light, pedestrians suddenly rushed out special scenes from the side can make a reasonable judgment, especially in the pedestrian when partially occluded, large items to carry, for accurate detection, and can reduce the snow, the ground reflection effect on the test results, maintaining high accuracy.(2) high detection speed: by optimizing algorithms, reducing the running time, thereby improving detection rate per unit of time.

Image processing and pattern recognition algorithm for learning->-> pedestrian detection method and Matlab simulation results analysis->OpenCV learning design and implementation of-> system-> system tests and summary.

Pedestrian detection is intended according to the video or picture information, image processing and computer vision algorithms, judging from the image whether or not pedestrians, pedestrians find the location and size, and furthermore identify pedestrian movements and gestures. Paper discussions around these specific goals step by step.First the realization and improvement of pedestrian detection algorithm based on gradient orientation histogram. This problem is caused by the actual demand for the introduction of intelligent video surveillance systems. Pedestrian detection module is the core and focus of video surveillance systems, intelligent analysis of abnormal alarm base. This requires pedestrian detection algorithm requires a high level of accuracy. Also need algorithms had very high speeds to achieve real-time monitoring of requests. Along with these requirements, we at the three algorithms are described in this chapter: HOG pedestrian recognition algorithms; based on cascade classifier $\mathrm{HOG}$; multiple support vector machine algorithm based on a fixed window[12]. Our new method is created with multiple support vector machine theory, combined with effective integration of fixed observation window redrawing the gradient orientation histogram, making identification algorithm of speed of a train speeds past has significantly improved. And then the object context associated behavioural model is, if we were to 
improve, not limited to just identify the pedestrians to walk upright, for example, are doing relatively large sport. So we put conditions to qualify in six categories of sports activities, the people pose can become very much. Our approach at this time is the result of using object recognition. Six sports were we qualified so limited the scope of object recognition, While the active type of fixing is relatively limited posture. Types of activities may be cited, specific objects, and body posture, limited State can train a Bayesian stochastic networks. Training using the new boundary of parameter estimation. And in the end achieved a very good result.Concludes with a context model of human relations. We propose a whole parts identification, respectively, global and local linkages between amendment, partial recognition results to verify the overall recognition accuracy of overall recognition result can limit the range of partial recognition. When a local recognition and the overall recognition conclusions coincide with greater positive that both sides are right. When partial recognition and overall recognition of conclusions contrary to each other when they believe that there is no finding optimal solutions. The main line of this article is that with the improvement of pedestrian detection system requirements, the expansion of the scope of application, step by step to find a solution, and in the process made some theoretical and practical results on the system.

\section{Conclusion}

With the rapid growth of the national economy and social progress, banking, power, transportation, and military installations in areas such as increasing demand for security alarm systems, application and development of intelligent video surveillance technology has put forward higher requirements. Pedestrian detection and pattern-recognition technology is an important trend of intelligent video surveillance technology to help security personnel found and dealt with, no one around the clock monitoring of abnormal behavior is important. A typical pedestrian detection and identification system includes a pedestrian detection and shadow elimination, human tracking and behaviour recognition module. This paper focuses on moving pedestrian detection based on background subtraction, simple target shadow removal and pedestrian behavior recognition technology and its application in three areas.

Moving target detection algorithms are the basis of pedestrian detection and identification technology. On frame difference method of target detection and detailed studies on background subtraction method, CW4 model proposed a theory based on background subtraction algorithm for moving target detection. This algorithm combines the advantages of frame difference method and background subtraction, and make full use of the chromatic aberration color and brightness of the image information, make test results more accurate. Experimental results show that the algorithm on the premise of guaranteeing real-time requirements, can significantly improve the accuracy of detection.

To more accurately detect the target, for shadow removal of moving object technologies are researched. Shaded images are analyzed in detail the characteristics and morphological characteristics of background and foreground pixels, color images and grayscale images, design based on background color is computed based on the weighted model and calculating models for shadow of the Division. Experimental results show that two shadow removal algorithm can efficiently remove shadows.

Pedestrians pedestrian crosses the virtual line and gesture recognition, virtual statistics such as the number of technology within the region studied. According to characteristics of binary image, puts a pedestrian gesture recognition algorithm based on template. Based on the initial position of 
pedestrians and the relationship between the current position and the virtual line presented a pedestrian-based location algorithm to determine whether it crosses the virtual line; in the cross line detection algorithm based on the statistical number of pedestrians in a virtual area of features.

Research of pedestrian detection and shadow elimination and behavior recognition algorithms in intelligent video surveillance, intelligent transport and navigation areas play an important practical and theoretical significance.

\section{Acknowledgements.}

This work was supported by Undergraduate Students Innovative Training Program of Jiangsu Province (201313984001Y). NingXu, student ID Number: 1116406026, currently is an undergraduate student of Applied Technology College of Soochow University. This work was directed by Yong Ren. Corresponding author is Yong Ren: renyong@ suda.edu.cn.

\section{Reference}

[1]. D. Geronimo, and A. M.Lopez. Vision-based Pedestrian Protection Systems for Intelligent Vehicles, BOOK, 2014.

[2]. P.Dollar, C. Wojek,B. Schiele, et al. Pedestrian detection: an evaluation of the state of the art [J].IEEE Transactions on PatternAnalysis andMachine Intelligence, 2012, 34(4): 743-761.

[3]. M. Enzweiler, and D.Gavrila. Monocular pedestrian detection: survey and experiments [J]. IEEE Transactions on Pattern Analysis andMachine Intelligence, 2009, 31(12): 2179-2195.

[4]. D. Geronimo, A. M.Lopez and A. D. Sappa, et al. Survey of pedestrian detection for advanced driver assistance systems [J]. IEEE Transactions on Pattern Analysis and Machine Intelligence, 2010, 32(7): 1239-1258.

[5]. M. Gavrila D, Munder S. Multi-cue Pedestrian Detection and Tracking from a Moving Vehicle[J]. International Journal of Computer Vision, 2007, 73:41-59. DOI:10.1007/s11263-006-9038-7.

[6]. Xu F, Liu X, Fujimura K. Pedestrian detection and tracking with night vision[J]. Ranaon on NllgnRanoraonYm, 2005, 6(1):63 - 71.

[7]. M. Gavrila D. Pedestrian Detection from a Moving Vehicle[J]. Lecture Notes in Computer Science, 2000:37-49.

[8]. Kang S, Lee H B A S. Real-Time Pedestrian Detection Using Support Vector Machines[J]. Lecture Notes in Computer Science, 2002:273-281.

[9]. Dollar P, Wojek C, Schiele B. Pedestrian Detection: An Evaluation of the State of the Art[J]. Pattern Analysis and Machine Intelligence, IEEE Transactions on, 2012, 34:743 - 761.

[10].Broggi A, Cerri P, Ghidoni S, et al. A New Approach to Urban Pedestrian Detection for Automatic Braking[J]. Intelligent Transportation Systems, IEEE Transactions on, 2009, 10(4):594 - 605.

[11].Cao X, Qiao H, Keane J. A Low-Cost Pedestrian-Detection System With a Single Optical Camera[J]. Ranaon on NllgnRanoraonYm, 2008, 9(1):58 - 67.

[12].David Gerónimo A L A A D S. Computer Vision Approaches to Pedestrian Detection: Visible 
Spectrum Survey[J]. Lecture Notes in Computer Science, 2007:547-554. 